\title{
Safety and efficacy of percutaneous nephrolithotomy in the Galdakao - Modified Supine Valdivia position: A prospective analysis
}

\author{
Luigi Quaresima, Alessandro Conti, Alexia Vici, Marco Tiroli, Daniele Cantoro, Admena Rreshketa, \\ Emanuele Principi, Matteo Tallè, Luca Moroni, Mahmoud Yehia, Giovanni Muzzonigro \\ Department of Clinical and Specialist Sciences, Division of Urology Polytechnic University of the Marche Region Medical School, Ancona, Italy. \\ Introduced as Poster at the $14^{\text {th }}$ National IEA Congress 26-28/02/2015, Milan, Italy.
}

\begin{abstract}
Summary Objective: Aim of the present study was to evaluate the safety and efficacy of Percutaneous Nephrolithotomy (PCNL) in the GaldakaoModified Supine Valdivia (GMSV) position in order to predict operative time, stone-free rate and onset of complications taking into account comorbidity, stone-related parameters and anatomic upper urinary tract abnormalities.

Material and methods: A prospective evaluation of patients who underwent to PCNL in GMSV position for renal stones > $2 \mathrm{~cm}$, from January 2009 to February 2015 was performed. According to the technique, upper urinary tract abnormalities, stone chemical and morphological characteristics, and patients' history were matched with operative outcome, in terms of stone-free, intervention time and incidence of perioperative complications.

Results: Seventy-two cases were collected; mean operative time was 105 minutes (DS 41): staghorn stones and the presence of comorbidity resulted statistically significant variables. The complication-rate resulted in line with data showed in literature: hyperpyrexia and hemorrhage were the more frequently complications found. The overall stone-free was reached in 48 patients (67\%), and it was influenced by patients' anatomic abnormalities.

Conclusions: In the treatment of renal stones, PCNL may be a safe and effective choice; nevertheless, patients' anatomic abnormalities or staghorn-stones may influence the outcomes. Thus, a prospective study with a larger population is needed to verify our outcomes.
\end{abstract}

KEY WORDS: Stones; Percutaneous Nephrolithotomy (PCNL); Stone-free Rate; Staghorn Stones; Complications.

Submitted 1 October 2015; Accepted 4 December 2015

\section{INTRODUCTION}

Percutaneous nephrolithotomy (PCNL) is an effective choice in the treatment of large kidney stones because of excellent outcomes and acceptable low morbidity (1). Standard treatment introduced in the 80's consists in percutaneous puncture of the lower calyx of the kidney, with subsequent progressive dilatation and positioning of an operative channel, a rigid or flexible nephroscope can be passed through, in order to perform the intrarenal lithotripsy. This access was originally obtained with patients set in a prone (2) position, in order to obtain a marked ventralization of the peritoneal structures and minimize the risk of accidental bowel perforation. The possibility of a supine position was first reported by Valdivia Uria et al. (3) in 1998, with the purpose of allowing an easier management of the airways from the anestesiologic side. Moreover, this position allows to the surgeon to perform a combined access to the upper urinary tract in an anterograde-retrograde fashion (Endoscopic Combined IntraRenal Surgery-ECIRS) (4). Currently a standardization for this procedure is missing, many centers still adopting the original standard prone position, while the use of GSMV is, in our opinion limited by concerns about the surgical advantages and the increased difficulty for the surgeon to achieve an appropriate access to the inferior calyces compared to the standard technique.

Aim of our study is to evaluate the advantages of the procedure in terms of efficacy (capacity of obtaining a complete removal of the stone burden - stone-free) and reduction of intra- and postoperative complications at our Department.

\section{MATERIAL AND MEthods}

We prospectively collected clinical data from patients treated at our Institution with PCNL in GSMV position for renal stone disease between January 2011 and February 2015. The following clinical variables at intervention were considered for the purposes of the study: gender, age, comorbidities, urine status and possible antibiotic therapy.

According to radiologic findings, also the following characteristics of the urinary tract were recorded: wideness of calyx and renal ampulla and presence of anomalies of kidney vessels or voluminous renal cysts, as shown by the pre-operative abdomen CT.

As for characteristics of the lithiasis, stone burden and number and radio-opacity were the main features considered. The post-operative outcomes were evaluated according to the following characteristics: duration of the intervention, complete stone-free, intra- or postoperative complications. Patients' follow up, conducted with radi-

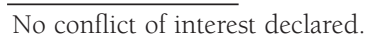


ological, clinical and labor investigations, was at least 3 months. Age was considered as a dichotomic variable $(<65$ y vs $>65$ y). Group comparisons for continuous variables were performed by means of Mann-Whitney $\mathrm{U}$ test; all results were summarized with percentiles. Association of categorical variables were estimate with Fisher Yates' exact test. Statistical significance was fixed at the $5 \%$ level for all tests.

\section{RESULTS}

A total of 72 patients were treated during the period considered. Forty-seven of them were males. Mean age at intervention was 54 years. In 41 cases (57\%) patients had been referred to the intervention because of complete staghorn calculi; in 28 cases (39\%) intervention was performed because of 3 or more single stones. Radiopaque lithiasis was observed in 51 cases (71\%), while in all other cases partially opaque or radiolucent stones at abdomen -RX were observed.

Population's pre-operative characteristics are reported in detail in Table 1.

In the post-operative period, 38 patients presented with complications requiring prolonged hospitalization. In particular, for 12 cases (16\%) an early-postoperative anemia, treated with blood transfusion was reported; 5 patients $(6,9 \%)$ developed renal hematoma and 2 patients complicated UTIs. A list of recorded complications is reported in Table 2.

Mean duration of the procedure was 105.5 minutes ( \pm 41.3), with a significantly longer operative time in patients with single staghorn stones compared to the group with multiple stones (112 \pm 35.6 min vs $46.7 \pm$ $24.9, \mathrm{p}=0.043$ ), as showed in Figure 1. Presence of comorbidities resulted associated with longer operative times ( $p=0.03$ ), but no difference could be found between the different comorbidity types ( $p>0.05$ ) (Figure 2). Finally, no difference was found between the different age groups.

In 48 cases (67\%) complete removal of the stone burden (stone-free patients) could be achieved, while in 24 cases (33\%) multiple small residuals were identified at the first

\section{Figure 1.}

PCNL operative time in relation to the presence/absence of staghorn stones.

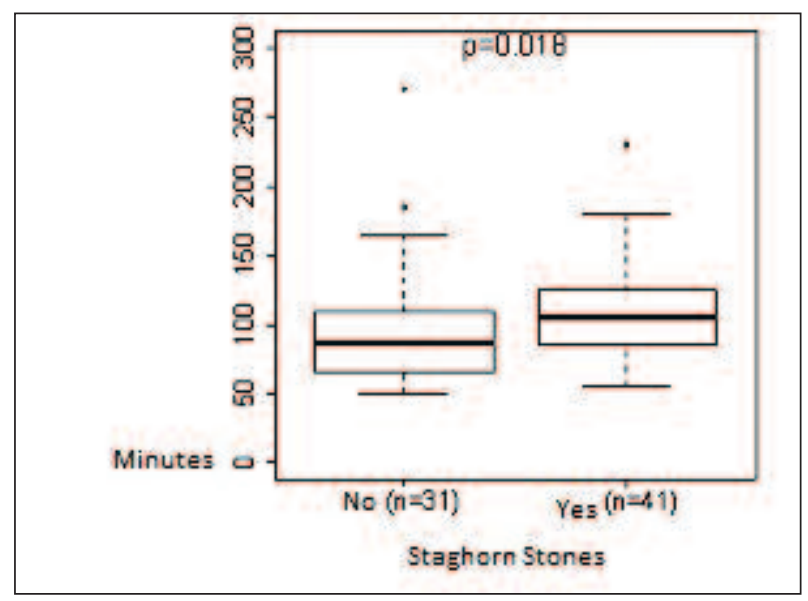

Table 1.

Pre-operative patients features.

\begin{tabular}{|lcc|}
\hline & N. patients & $\%$ \\
\hline Sex & 47 & 65 \\
$\mathrm{~F}$ & 25 & 35 \\
\hline Age & & \\
$<65$ years & 52 & 72 \\
$>65$ years & 20 & 28 \\
\hline Characteristic of the stone & & \\
Radiolucent & 13 & 18 \\
Partially opaque & 8 & 11 \\
Radiopaque & 51 & 71 \\
Single stone & 41 & 57 \\
Multiple stones & 28 & 39 \\
\hline Comorbidity & & \\
Hypertension & 25 & 35 \\
Ischemic heart disease & 13 & 18 \\
Chronic bronchitis & 6 & 8 \\
Diabetes & 15 & 21 \\
Chronic renal failure & 4 & 6 \\
\hline
\end{tabular}

\section{Table 2.}

Post-operative PCNL complications.

\begin{tabular}{|lcc|}
\hline Complications & N. patients & $\%$ \\
\hline Anemia required blood transfusion & 12 & $16.6 \%$ \\
\hline Fever & 10 & $13.8 \%$ \\
\hline Renal hematoma & 5 & $6.9 \%$ \\
\hline Atrial fibrillation required cardioversion & 3 & $4.1 \%$ \\
\hline Peritoneal/pleuric effusion & 4 & $5.5 \%$ \\
\hline Persistent haematuria & 2 & $2.7 \%$ \\
\hline Complicated urinary tract infections & 2 & $2.7 \%$ \\
\hline
\end{tabular}

\section{Figure 2.}

PCNL operative time in relation to the comorbidity.

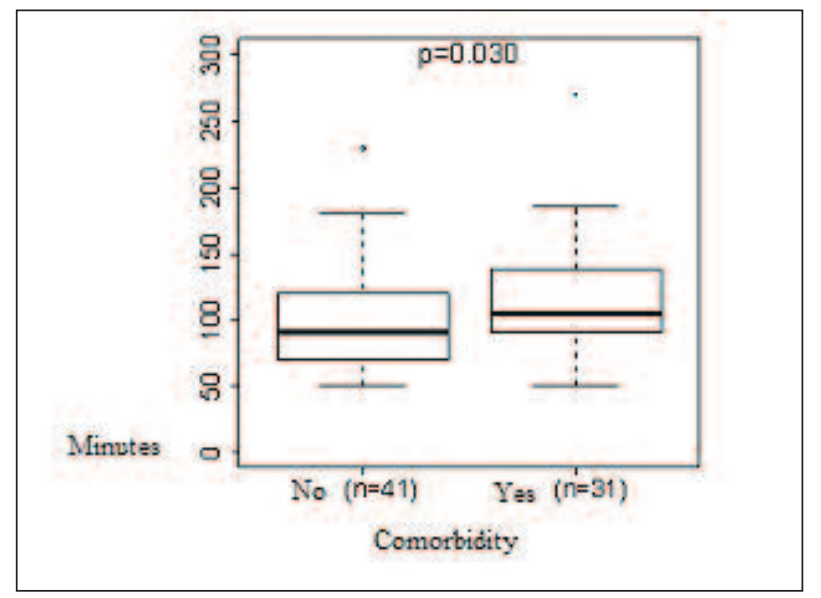

follow-up; in 10 cases $(13,8 \%)$ difficulties were to be ascribed to the presence of anatomic anomalies. The stone-free rate was therefore higher in patients without anatomical anomalies (94\% vs 6\%, p = 0.025), as highlighted in Figure 3. No significant associations were found between possibility of stone-free and type of stone (staghorn vs multiple lithiasis, $\mathrm{p}=0.215$ ). The difference in stone-free rate, according to the presence of staghorn calculi, is reported in Figure 4. 
Figure 3.

Influence of anatomical anomalies in the stone-free rate.

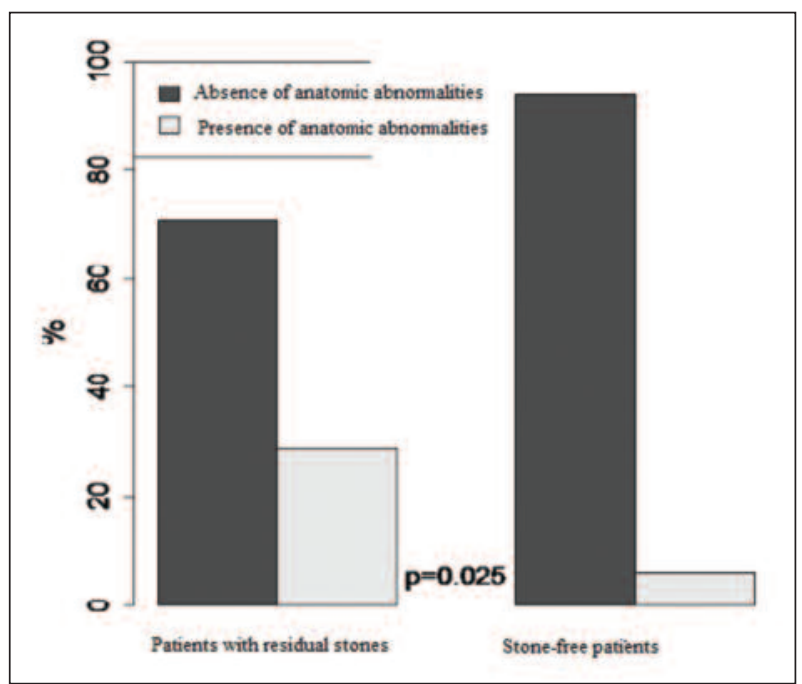

Figure 4.

Influence of staghorn stones in the stone-free rate.

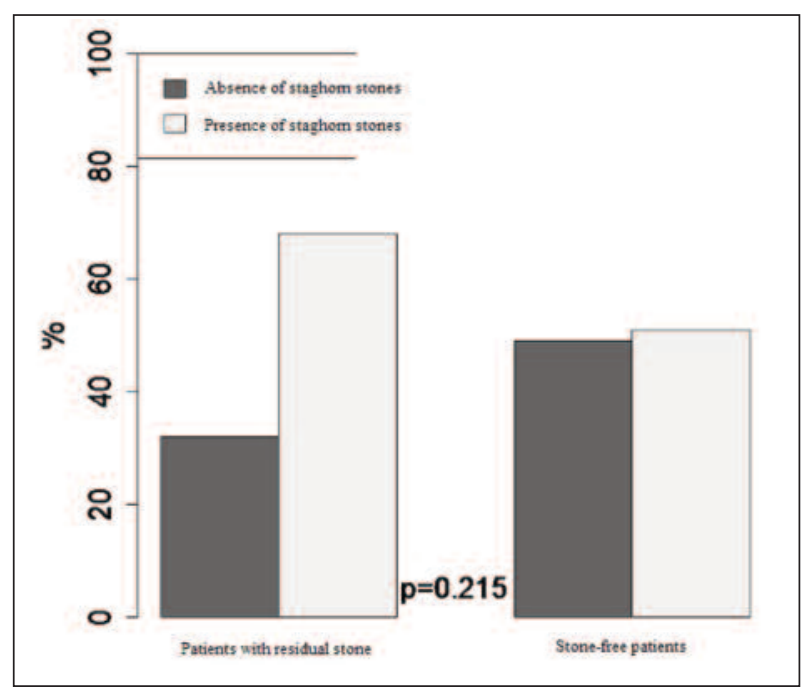

\section{Discussion}

Although PCNL is considered the gold standard treatment modality for large renal stones, this procedure is not lacking of side effects. In particular, damage to the large blood vessel and late hemorrhage are rare (less than $1 \%)$ but serious and potentially life-threatening complications of PCNL (5). Blood transfusion and prolonged hospital stay, or readmission due to the hemorrhage may occur, requiring extra costs on the health care system (6), Several authors have analyzed the variables associated to the perioperative hemorrhage: a prospective multicenter observational study leaded by De Fata et al. (7), on 397 supine PCNL procedure performed in 15 Spanish centers, showed how multiple percutaneous tracts and middle calix puncture have resulted the only statistically significant variables associated with the decrease in hemoglobin levels.

Positive preoperative urine culture or preexisting UTI were reported by several authors as a significant parameter predicting complications (8-10): according to other studies $(11,12)$ we have not founded this correlation with complications.

Few studies investigated the correlation between normal or impaired renal function and onset of complications with discordant results: Yaycioglu (13) reported no difference, while Sairam and Falahatkar $(14,15)$ showed a significantly increased complication rates associated to chronic kidney disease. In our study, only four patients were affected by impaired renal function pre PCNL, and with a postoperative progress in line with other patients. The association between anatomic upper urinary tract abnormalities and complication rates in patients undergone to PCNL was investigated by some authors. In particular, Osther (16) and Rais-Bahrami (17) demonstrated as alteration in position and axis of the kidney, renal morphology, vasculature and mobility of the kidney, morphology of calices, pelvis and upper ureter were statistically associated to a higher number of complications, according to our results.

Stone-related parameters (size, opacity, number, localization, staghorn and complex/multiple stones) resulted to be connected in our experience to a higher complication rate, in line with De la Rosette (18) and Akman's (19) studies. In the study leaded by Shin stone's size and staghorn or complex stone had a significant influence only in minor complications, such as UTI or pain (20). Some studies $(9,15)$, one of the most recent was published on February 2015 by Falahatkar et al. (15) reported the PCNL complications according to the modified Clavien system (MCC), a scale that allows to classify complications basing on life-treating condition, interventions required, and disability. Nevertheless the MCC cannot predict, as said by the same authors, the occurrence of a specific complication and cannot reveal its reason. Moreover, also due to different surgical managements, the sub classification of the higher Clavien grades has low reliability (15).

Regarding the mean operative time, our experience highlights a longer time than what it is usually presented in literature: comorbidity resulted statistically correlated to the length of the procedure instead of the patient's age.

The overall stone-free rate was reached in $67 \%$ of patients, in line with the data reported in litherature: recently Noureldin et al compared two different scoring system to predict stone-free status post percutaneous nephrolithotomy, Guy's versus S.T.O.N.E., highlighting how both systems were significantly associated with the estimated blood loss (21).

Interestingly several articles reported different results about the effect of operative time on complications ( 9 , 11, 15, 18, 20): Labate et al. found that prolonged operative time has significantly predicted a higher risk of major complication, such as bleeding requiring blood transfusion or impaired renal function while Falahtakar et al. reported no correlation.

Our study presents some limitations: although we included all cases during the period of the study in order to complete a prospective evaluation, patients' number have not reached the specimen analyzed in other articles. Moreover, the procedure included in our study were per- 
formed by two different operators, even though both surgeons had a long-standing experience in percutaneous nephrolithotomy. Therefore, a prospective randomized study with a larger population is needed to confirm our observations.

\section{Conclusion}

In conclusion, PCNL in GSMV may be a safe and effective choice to treat large renal stones.

Nevertheless, patients' anatomic abnormalities may influence stone-free rate while the presence of comorbidity or a staghorn stone may increase operative time. Thus, a prospective study with a larger population is needed to verify our outcomes.

\section{REFERENCES}

1 Segura JW, Patterson DE, LeRoy AJ, et al. Percutaneous lithotripsy. J Urol. 1983; 130:1051-4.

2. Alken P, Hutschenreiter G, Gunter R, et al. Percutaneous stone manipulation. J Urol. 1981; 125:463-466.

3. Valdivia Uria JG, Valle Gerhold J, Lopez Lopez JA, et al. Technique and complications of percutaneous nephroscopy: experience with 557 patients in the supine position. J Urol 1998; 160:1975-8.

4. Ibarluzea G, Scoffone CM, Cracco CM, et al. Supine Valdivia and modified lithotomy position for simultaneous anterograde and retrograde endourological access. BJU Int. 2007; 100:233-236.

5. Basiri A, Tabibi A, Nouralizadeh A, et al. Comparison of safety and efficacy of Laparoscopic Pyelolithotomy versus Percutaneous Nephrolithotomy in patients with renal pelvic stones: a randomized clinical trial. Urol J. 2014; 11:1932-7.

6. Stuard Wolf J. Urinary Lithiasis: Percutaneous approaches to the upper urinary tract collecting system. In Wein AJ, Kavoussi LR Campbell-Walsh Urology 10th ed. Philadelphia, Elsevier; 2012; p.1348-54.

7. Ramón de Fata F, Pérez D, Resel-Folkersma L, et al. Analysis of the factors affecting blood loss in percutaneous nephrolithotomy: a registry of the Spanish Association of Urology in the supine position. Actas Urol Esp. 2013; 37:527-32.

8. El-Nahas AR, Eraky I, Shokeir AA, et al. Factors affecting stonefree rate and complications of percutaneous nephrolithotomy for treatment of staghorn stone. Urology. 2012; 79:1236-41.

9. Palmero JL, Nuño de la Rosa I, Miralles J, et al. Study of predictive factors for complications after percutaneous nephrolithotomy

\section{Correspondence}

Luigi Quaresima (Corresponding Author)

luigiquaresima@yahoo.it

Department of Clinical and Specialist Sciences, Division of Urology

Polytechnic University of the Marche Region Medical School

Via Conca 71 - 60100 Ancona, Italy

Alessandro Conti

Alexia Vici

Marco Tiroli

Daniele Cantoro

Admena Rreshketa

Emanuele Principi

Matteo Tallè

Luca Moron

Mahmoud Yehia

Giovanni Muzzonigro according to the Clavien Classification. Actas Urol Esp. 2013; 37:412-8.

10. Olbert PJ, Hegele A, Schrader AJ, et al. Pre- and perioperative predictors of short-term clinical outcomes in patients undergoing percutaneous nephrolitholapaxy. Urol Res. 2007; 35:225-30.

11. Labate G, Modi P, Timoney A, et al. The percutaneous nephrolithotomy global study: Classification of complications. J Endourol. 2011; 25:1275-80.

12. Onal B, Dogan HS, Satar N, et al. Factors affecting complication rates of percutaneous nephrolithotomy in children: Results of a multi-institutional retrospective analysis by the Turkish pediatric urology society. J Urol. 2014; 191:777-82.

13. Yaycioglu O, Egilmez T, Gul U, et al. Percutaneous nephrolithotomy in patients with normal versus impaired renal function. Urol Res. 2007; 35:101-105.

14. Sairam K, Scoffone CM, Alken P, et al. Percutaneous nephrolithotomy and chronic kidney disease: results from the CROES PCNL Global Study. J Urol. 2012; 188:1195-200.

15. Falahatkar S, Moghaddam KG, Kazemnezhad E, et al. Factor affecting complications according to the modified Clavien classification in complete supine percutaneous nephrolithotomy. Can urol Assoc J, 2015; 9:e83-92.

16. Osther PJ, Razvi H, Liatsikos E, et al. Percutaneous nephrolithotomy among patients with renal anomailes: patients characteristics and outcomes; a subgroup analysis of the clinical research office of the endourological society global percutaneous nephrolithotomy study. J Endourol. 2011; 25:1627-32.

17. Rais-Bahrami S, Friedlander JI, Duty BD, et al. Difficulties with access in percutaneous renal surgery. Ther Adv Urol. 2011; 3:59-68.

18. De la rosette JJ, Zuazu JR, Tsakiris P, et al. Prognostic factors and percutaneous nephrolithotomy morbidity: A multivariate analysis of a contemporary series using the Clavien Classification. J Urol. 2008; 180:2489-93.

19. Akman T, Binbay M, Akcay M, et al. Variables that influence operative time during percutaneous nephrolithotomy: an analysis of 1897 cases. J Endourol. 2011; 25:1269-73.

20. Shin TS, Cho HJ, Hong SH, et al. Complications of percutaneous nephrolithotomy classified by the modified Clavien grading system: a single center's experience over 16 years. Korean J Urol. 2011; 52:769-75.

21. Noureldin YA, Elkoushy MA, Andonian S. Which is better? Guy's versuS S.T.O.N.E. nephrolithometry scoring systems in predicting stone-free status post-percutaneous nephrolithotomy. World J Urol. 2015; 33:1821-5. 